5 - ORIGINAL ARTICLE

ISCHEMIA-REPERFUSION

\title{
The role of bradykinin in lung ischemia-reperfusion injury in a rat lung transplantation model $^{1}$
}

\author{
Zheng Tang', Zhiwei Wang ${ }^{\mathrm{II}}$, Zhipeng Hu${ }^{\mathrm{III}}$, Min Zhang ${ }^{\mathrm{III}}$, Luocheng $\mathrm{Li}^{\mathrm{III}}$, Bowen $\mathrm{Li}^{\mathrm{IV}}$ \\ DOI: http://dx.doi.org/10.1590/S0102-865020160120000005 \\ ${ }^{\mathrm{I}} \mathrm{PhD}$, Department of Cardiovascular Surgery, Renmin Hospital, Wuhan University, China. Conception and design of the study, acquisition and \\ interpretation of data, manuscript writing. \\ ${ }^{\mathrm{II}} \mathrm{PhD}$, Full Professor, Department of Cardiovascular Surgery, Renmin Hospital, Wuhan University, China. Design and supervised all phases of the \\ study, critical revision. \\ III PhD, Department of Cardiovascular Surgery, Renmin Hospital, Wuhan University, China. Acquisition of data, critical revision. \\ IV Master, Department of Cardiovascular Surgery, Renmin Hospital, Wuhan University, China. Acquisition of data.
}

\section{ABSTRACT}

PURPOSE: To investigate the role of bradykinin in a rat lung transplantation (LTx) model and preliminarily discuss the relationship between bradykinin and CD26/DPP-4.

METHODS: Rats were randomly divided into four groups: Control (CON), Sham, low potassium dextranglucose (LPD), and AB192 ( $n=15$ /group). Orthotopic single LTx was performed in the LPD and AB192 groups. The donor lungs were flush-perfused and preserved with low potassium dextranglucose (LPD) or LPD+CD26/DPP-4 catalytic inhibitor (AB192). LTx was performed after $18 \mathrm{~h}$ cold ischemia time and harvested two days post-LTx. Blood gas analysis $\left(\mathrm{PO}_{2}\right)$, wet/dry weight ratio (W/D), myeloperoxidase activity (MPO), and lipid peroxidation (MDA) were analyzed at $48 \mathrm{hr}$ after transplantation. Immunohistochemical (IHC) analysis was performed in the same sample and validated by Western-Blot.

RESULTS: Compared to the LPD group, the AB192 group showed higher $\mathrm{PO}_{2}$, lower W/D ratio, and decreased MPO and MDA. IHC studies showed strong bradykinin $\beta 2$ receptor (B2R) staining in the LPD group, especially in inflammatory cells, alveolar macrophages, and respiratory epithelial cells. Expression of B2R by Western-Blot was significantly different between the AB192 and LPD groups.

CONCLUSION: Bradykinin may be a competitive substrate of DPP-4, and decreased bradykinin levels may enhance protective effects against ischemia/reperfusion injury during LTX.

Key words: Receptor, Bradykinin B2. Lung Transplantation. Reperfusion Injury. Ischemia. Rats 


\section{Introduction}

Bradykinin is regarded as a substrate of the CD26/DPP-4 enzyme ${ }^{1}$. Research has revealed that bradykinin B2 receptor (B2R) antagonists play a protective role against ischemia/reperfusion (I/R) injury, particularly in transplantation ${ }^{2-5}$, but the significance of bradykinin in IR and the relationship between bradykinin and CD26/DPP-4 are not completely understood. It is accepted that the kallikrein-kinin system expresses potent biological activities through its final product, bradykinin ${ }^{6}$, and that bradykinin acts via two receptor subtypes. The bradykinin $\mathrm{B} 1$ receptor $(\mathrm{B} 1 \mathrm{R})$ is up-regulated under inflammatory conditions, whereas $B 2 R$ is constitutively expressed ${ }^{7}$. Some studies have shown that B1R modulates the late phase of lung inflammation ${ }^{8,9}$, while $\mathrm{B} 2 \mathrm{R}$ is involved in full-stage airway responses, and mediates NF-kB activation in airway epithelial cells ${ }^{10}$. Identifying the bradykinin receptor subtypes involved at different stages during inflammation of the lung is crucial to improve our understanding of complex pulmonary conditions, including treatment (Tx)-induced lung I/R injury.

Primary graft dysfunction (PGD) following lung transplantation (LTx) has been described as an acute lung injury syndrome within $72 \mathrm{hr}$ of transplantation that is triggered by marked I/R injury, and is associated with pulmonary edema, severe hypoxemia, bilateral pulmonary infiltrates, and other conditions. Importantly, all symptoms have been shown to occur no later than $72 \mathrm{hr}$ after $\mathrm{LTx}^{11,12}$. The treatment for PGD remains unacceptable: patients undergoing LTx with PGD have a mortality rate up to $60 \%{ }^{13}$. It has been demonstrated ${ }^{14}$ that perfusion with a specific inhibitor of DPP-4 significantly decreases the incidence and severity of pulmonary PGD and improves recovery after LTx. In addition, the severity of PGD was shown to be related to the time of cold ischemia ${ }^{14,15}$. Extended cold ischemia time better reflects the role of the target protein, further broadening the scope of CD26/DPP -4 inhibitors for potential therapeutic use.

As a proof of concept approach, and due to the timing of PGD, we chose to investigate the role of $B 2 R$ in $I / R$ after transplantation. Next, we established a rat LTx model to investigate the role of bradykinin in PGD and preliminarily discuss the relationship between bradykinin and CD26/DPP-4. Finally, we ascertained whether the severity of $\mathrm{I} / \mathrm{R}$ injury correlates with augmentation of B2R.

\section{Methods}

The animal protocol was approved by the Animal Ethics
Review Committee of Wuhan University and the studies were performed in compliance with the Principles of Laboratory animal care.

Orthotopic left LTx was performed in healthy male adult Sprague Dawley (SD) rats (270 - $300 \mathrm{~g}$ ) using a cuff technique for anastomosis. Rats were randomized into four groups $(n=15$ per group): control (CON), sham operated (Sham), LPD, and LPD + AB192 groups. The CON group did not undergo the operation. The Sham group received tracheal intubation and a thoracotomy. Orthotopic left LTx was performed in the LPD and AB192 groups. A separate group of 30 rats served as donors. The lungs were flush-perfused and preserved with LPD or with the LPD+DPP-4 catalytic inhibitor AB192. LTx was performed after $18 \mathrm{~h}$ cold of ischemia time and harvested at 2 days.

At harvest, blood oxygenation was measured. Graft tissues were taken for wet/dry (W/T) weight ratio, myeloperoxidase (MPO) activity, and lipid peroxidation (MDA) measurement, and for further examination.

\section{Donor procedure}

The donors were anesthetized by intraperitoneal injection of $0.2 \%$ pentobarbital $(50 \mathrm{mg} / \mathrm{kg})$ and orotracheally intubated with a 14-gauge intravenous catheter. The tube was connected to a pressure controlled ventilator (Model 683 Harvard Rodent Ventilator, Harvard Apparatus, South Natick, MA) and the lungs ventilated a fraction of inspired oxygen of one, a tidal volume of $10 \mathrm{~mL} / \mathrm{kg}$ at 75 breaths/min, and a positive end-expiratory pressure of $2.5 \mathrm{~cm} \mathrm{H} \mathrm{H}_{2} \mathrm{O}$. After a median laparosternotomy, $1000 \mathrm{IU} / \mathrm{kg}$ of heparin was injected into the inferior vena cava. The lungs were flushed by the pulmonary artery (PA) with $20 \mathrm{~mL}$ of LPD at $4^{\circ} \mathrm{C}$ in the CON group, and with $20 \mathrm{~mL}$ LPD+AB192 (final concentration $25 \mathrm{~mol} / \mathrm{L}$ ) in the experimental groups. With the lungs inflated, the heart-lung-block was explanted. The left lung was isolated and stored at $4^{\circ} \mathrm{C}$ with either LPD or LPD+AB192.

\section{Recipient procedure}

Anesthesia, intubation, and ventilation were applied to the recipients as described above. All three structures (PV, PA, and main bronchus) were clamped and incised, and the cuffed respective donor structures inserted and fixed using a 6-0 nylon ligature. The transplanted lung was inflated, the thoracotomy closed, and the lung extubated. For the first two postoperative days, recipients received a standardized regimen of analgesic treatment. 


\section{Graft assessment}

At the time of harvest, graft oxygenation was evaluated by sampling blood directly from the PV of the transplanted lung. The graft was harvested, divided into thirds, and stored at $-80^{\circ} \mathrm{C}$ for further studies.

\section{Wet/dry weight ratio}

One part of each harvested lung was weighed and placed in an oven at $180^{\circ} \mathrm{C}$ for $48 \mathrm{hr}$. The portion was reweighed, and the ratio of the weight before and after drying was calculated. This assay reflects lung fluid content, indicating the extent of post reperfusion pulmonary edema.

\section{MPO activity assay}

Quantitative MPO activity was determined by measuring neutrophil migration into the graft. Lung tissue was homogenized with a tissue/buffer ratio of $50 \mathrm{mg} / \mathrm{mL}$ and absorbance was immediately measured at $450 \mathrm{~nm}$. Enzyme activity was expressed as unit per gram of tissue protein $(\mathrm{U} / \mathrm{g})$.

\section{Estimation of lipid peroxidation}

This assay is based on the formation of a complex between malondialdehyde (MDA) and two molecules of thiobarbituric acid in an acid medium. MDA concentration is determined by measuring the absorbance at $532 \mathrm{~nm}$ and is expressed in $\mu \mathrm{M}$.

\section{Immunohistochemical staining for $B 2 R$}

The tissue sections were incubated with rabbit polyclonal antibody to B2R according to manufacturer's instructions. Primary and biotinylated secondary antibody (horseradish peroxidase, HRP) binding were visualized by 3,30-diaminobenzidine. All cells were examined and classified as positive (brown-stained) or negative (not stained) for B2R.

\section{Western blotting}

Western blotting analysis was performed as routinely described. In brief, the protein was homogenized and separated by electrophoresis. The membrane was blocked and incubated with a rabbit polyclonal antibody to $\mathrm{B} 2 \mathrm{R}$, and subsequently incubated with a HRP secondary antibody (Abcam, Cambridge, UK). $\beta$-actin
(Epitomics, Burlingame, A) was chosen as the loading control. The relative density of proteins to $\beta$-actin was analyzed with Odyssey (LI-COR, Lincoln, NE).

\section{Reagents and drugs}

The following drugs/kits were used: AB192 (bis(4acetamidophenyl)1-(S)-prolylpyrrolidine-2(R,S)-phosphonate, University Hospital Zurich, Zurich, Switzerland); LPD (Gibco Invitrogen, Grand Island, US); myeloperoxidase Assay Kit (ab111749, Abcam, Cambridge, UK); Lipid Peroxidation (MDA) Assay (ab118970, Abcam, Cambridge, UK); B2R rabbit polyclonal antibody (orb13265, Biorbyt Ltd, Cambridge, UK).

\section{Statistical analysis}

Statistical analysis was performed using GraphPad Prism (Version 6.01 for Windows) statistical software. Results are expressed as mean $\pm \mathrm{SD}$. Student $t$ tests were used to compare the significant differences between groups. Statistical significance was set at $p<0.05$.

\section{Results}

\section{Oxygenation}

At $48 \mathrm{hr}$ post-treatment, oxygenation capacity was significantly higher in the AB192 group versus the LPD group $(125.70 \pm 2.90 \mathrm{mmHg}$ vs. $92.33 \pm 2.62 \mathrm{mmHg}, p<0.05)$. There was no difference between the CON and Sham groups (496.50 \pm 2.59 $\mathrm{mmHg} v s .489 .90 \pm 2.17 \mathrm{mmHg}, p>0.05$ ). In addition, oxygenation capacity was significantly higher in the CON and Sham groups compared to the AB192 group $(p<0.05)$, suggesting improved ventilation function with preconditioning of a graft specific DPP-4 inhibitor (Figure 1).

\section{Wet/dry weight ratio}

Tissue edema was significantly less in the AB192 group versus the LPD group $(6.46 \pm 0.26 v s .8 .55 \pm 0.28, p<0.05)$. There was no difference between the CON and Sham groups (5.17 \pm 0.14 vs. $5.40 \pm 0.14, p>0.05)$. Tissue edema was significantly less in the CON and Sham groups compared to the AB192 group $(p<0.05$; Figure 1). 

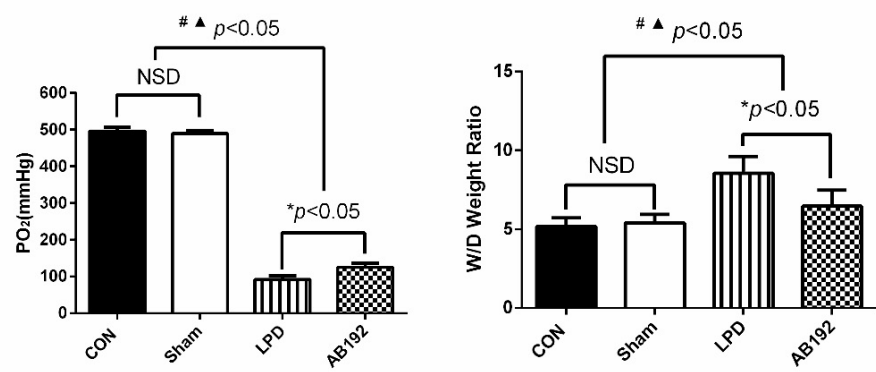

FIGURE 1 - $^{*} p<0.05$ LPD $v s$. AB192. $" p<0.05$ AB192 vs. CON. ${ }^{\star} p<0.05$ AB192 vs. Sham. There were no significantly differences (NSD) between the CON group and the Sham group $(p>0.05)$.

\section{MPO activity}

At harvest, MPO was significantly lower in LPD group compared to the AB192 group $(1.91 \pm 0.12 \mathrm{U} / \mathrm{g} v s .3 .38 \pm 0.12 \mathrm{U} / \mathrm{g}$, $p<0.05$ ), suggesting that MPO-decline was significant

in the AB192-perfused group. There was no difference between the CON and Sham groups $(0.95 \pm 0.08$ $\mathrm{U} / \mathrm{g} v s .1 .16 \pm 0.09 \mathrm{U} / \mathrm{g}, p>0.05)$. Moreover, MPO was lower in the CON and Sham groups compared to the AB192 group $(p<0.05)$. These findings suggest that the expression of bradykinin might be associated with myeloid differentiation, and related to decreases or increases in inflammation (Figure 2).

\section{Estimation of lipid peroxidation}

Forty-eight hours after graft reperfusion, significantly lower MDA was observed in the LPD group compared to the AB192 group $(1.97 \pm 0.14 \mu \mathrm{M} v s .3 .04 \pm 0.14 \mu \mathrm{M}, p<0.05)$. There was no difference between the CON and Sham groups $(0.94 \pm 0.09$ $\mu \mathrm{M} v s .1 .05 \pm 0.09 \mu \mathrm{M}, p>0.05)$. Moreover, MPO was significantly lower in the CON and Sham groups compared to the AB192 group $(p<0.05)$. Based on these data, we can estimate the degree of lipid peroxidation in pulmonary samples after LTx. In addition, the results of MDA represented different degrees of cytotoxicity and oxidative stress to determine whether or not to treatment with AB192 (Figure 2).
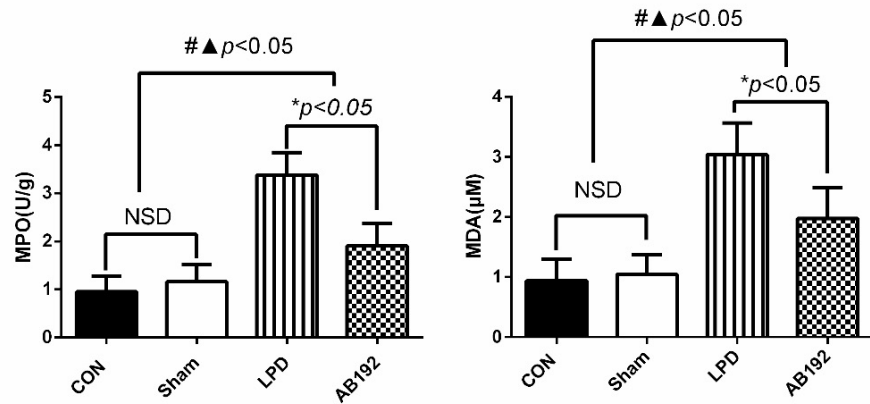

FIGURE $2-p<0.05$ LPD $v s$. AB192. ${ }^{*} p<0.05$ AB192 vs. CON. ${ }^{\star} p<0.05$ AB192 vs. Sham. There were no significantly differences (NSD) between the CON group and the Sham group ( $p>0.05)$.

\section{Immunohistochemistry evaluation}

Comparing histologic sections at $48 \mathrm{hr}$ after reperfusion, grafts from the LPD group showed stronger positive staining in the lungs, inflammatory cells, alveolar macrophages (AM), and respiratory epithelial cells versus grafts from the AB192 group. Conversely, grafts from the CON and Sham groups showed relatively negative staining. As shown in Figure 3, there were more inflammatory cells in the Sham group compared to the CON group. Staining in the AM was only slightly visible.

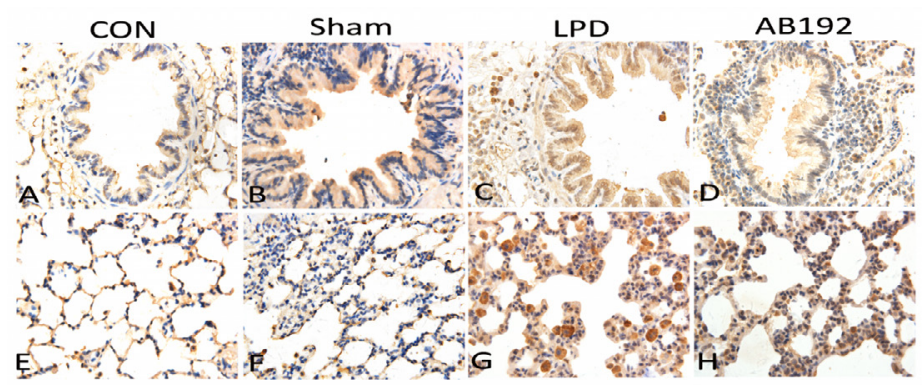

FIGURE 3 - Grafts from CON (A,E) and Sham (B,F) showed a negatively staining with $\mathrm{B} 2 \mathrm{R}$ in inflammatory cells and REC, compared with LPD and AB192 groups. More inflammatory cells in the Sham group,comparing with $\mathrm{CON}, \mathrm{AM}$ in the two groups were barely found. Representative sections of REC and inflammatory cells stained with B2R show strongly positive in the LPD group $(\mathbf{C})$ compared with the AB192 group (D), and AM showed obviously decreased B2R in the AB192 group (H) compared with the LPD group $(\mathbf{G})$. (magnification $\times 200$ ).

\section{Western blotting}

To verify the immunohistochemical results, we selected to investigate B2R for further analysis. The expression of B2R in the LPD and AB192 groups were significantly higher compared to 
the CON and Sham groups $(p<0.05)$, while the expression of B2R was significantly higher in the LPD group versus the AB192 group $(p<0.05)$. However, there was no difference in $\mathrm{B} 2 \mathrm{R}$ expression between the CON and Sham groups ( $p>0.05$ ) (Figure 4 ).

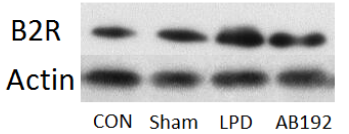

CON Sham LPD AB192

Discussion

Although many studies on DPP-4 have been published, and research has shown bradykinin to play a crucial role in $\mathrm{I} / \mathrm{R}$ injury, the relationship between bradykinin and DPP-4 in LTx with $I / R$ injury is still incompletely understood. In the current investigation, our aim was to preliminarily investigate this relationship, and to evaluate the role of bradykinin in the early stage of post-LTx I/R injury, specifically focusing on PGD with different expression of bradykinin. A Tx model was established using AB192, a graft-specific inhibitor of DPP-4 activity that can ameliorate PGD and promote gradual restoration of pulmonary function within a week ${ }^{14}$. The main findings from these studies are: 1) inhibition of CD26/DPP IV ameliorated PGD in the rat LTx model, protecting against ischaemia-reperfusion injury; 2) mild inflammation in the AB192 group; 3)expression of bradykinin was down-regulated with AB192 treatment, with sustained suppression of inflammation in the recipients; 4) decreased protein levels might be beneficial to attenuate the I/R injury after LTx; and 5) proper application of bradykinin may enhanced protective effects against I/R injury during transplantation.

Firstly, the current results verified the effects of a DPP-4 inhibitor on PGD after LTx, corroborating data reported by Zhai et $a l .{ }^{14}$. Secondly, research has shown that many inflammatory cells express B1R, including macrophages, epithelial cells, smooth muscle cells, endothelial cells and others ${ }^{16}$. While the main B1R-mediated effects include vasodilatation, plasma protein extravasation, pain mediation, activation of leukocyte- endothelial cell interactions, and leukocyte recruitment ${ }^{17}, \mathrm{~B} 2 \mathrm{R}$ is only involved in airway inflammatory responses. Similarly, we found that $\mathrm{B} 2 \mathrm{R}$ was also expressed in inflammatory cells including $\mathrm{AM}$, in addition to REC in the early phase of $\mathrm{I} / \mathrm{R}$ injury. Based on the MPO and IHC results, this might be attributable to the graft immune responses or related to Ras/Raf-1/ERK Pathway ${ }^{10}$, and may also represent B2R-mediated effects on various cells. Thirdly, the role of bradykinin in lung inflammation has been characterized by an early infiltration of neutrophils and vascular damage ${ }^{18}$. In contrast, DPP-4 is expressed on various epithelial, endothelial and lymphatic cellular surfaces ${ }^{19}$, and cleaves many potent biological proteins. Thus the argument that bradykinin is a substrate of DPP4 is not entirely confirmed. In our study, we found that bradykinin was down-regulated with DPP-4 inhibitor treatment, and therefore speculate that bradykinin is a competitive substrate of the DPP4 enzyme. Finally, DPP-4 inhibition increased local pulmonary bradykinin levels. This effect correlated with ventilatory function and pulmonary structural integrity, as well as PGD after LTx. In addition, injection of a B2R antagonist combined with a specific DPP-4 inhibitor, attenuated IRI in lung grafts and enabled lung recovery.

There are several limitations to this study. First, our findings were based on a single time point, which did not allow for dynamic observations. Second, Lewis rats may be better for our experimental animals than SD rats. Third, the rats which had graft failure were excluded. Fourth, this study was a preliminarily investigation and thus alternative mechanisms were not investigated.

\section{Conclusions}

Bradykinin may be a competitive substrate of DPP-4, and decreased bradykinin levels may provide protective effects against I/R injury during LTx. This study provides a preliminary examination and discussion on the role of bradykinin in PGD and the relationship between bradykinin and CD26/DPP-4

\section{References}

1. Mentlein R. Dipeptidyl-peptidase IV (CD26)--role in the inactivation of regulatory peptides. Regul Pept. 1999 Nov;85(1):9-24. PMID: 10588446.

2. Perron MS, Gobeil F Jr, Pelletier S, Regoli D, Sirois P. Involvement of bradykinin B1 and B2 receptors in pulmonary leukocyte accumulation induced by Sephadex beads in guinea pigs. Eur J Pharmacol. 1999 Jul;376(1-2):83-9. PMID: 10440093. 
3. Farmer SG, Wilkins DE, Meeker SA, Seeds EA, Page CP. Effects of bradykinin receptor antagonists on antigen-induced respiratory distress, airway hyperresponsiveness and eosinophilia in guineapigs. Br J Pharmacol. 1992 Nov;107(3):653-9. PMID: 1335332.

4. Mashito Y, Ichinose M, Shirato K. Bradykinin B2 antagonist HOE 140 inhibits late allergic microvascular leakage in guinea pig airways. Immunopharmacology. 1999 Sep;43(2-3):249-53. PMID: 10596860

5. Nowak K, Weih S, Post S, Gebhard MM, Hohenberger P. Bradykinin in ischemia-reperfusion injury of the rat lung. J Physio Pharmacol. 2007 Nov;58 Suppl 5(Pt 2):513-22. PMID: 18204165.

6. Oh-Ishi S. Biological regulation by the kallikrein-kinin system: a study with a kininogen -deficient rat strain. Nihon Yakurigaku Zasshi. 1993 Apr;101(4):209-18. PMID: 8514206.

7. Marceau F, Regoli D. Bradykinin receptor ligands: therapeutic perspectives. Nat Rev Drug Discov. 2004 Oct;3(10):845-52. PMID: 15459675.

8. El-Kady MM, Girgis ZI, Abd El-Rasheed EA, Shaker O, Attallah MI, Soliman AA. Role of selective blocking of bradykinin receptor subtypes in attenuating allergic airway inflammation in guinea pigs. Eur J Pharmacol. 2016 Oct 5;788:152-9. PMID: 27321873.

9. Gama Landgraf R, Sirois P, Jancar S. Differential modulation of murine lung inflammation by bradykinin B1 and B2 selective receptor antagonists. Eur J Pharmacol. 2003 Jan 26;460(1):75-83. PMID: 12535863.

10. Chen BC, Yu CC, Lei HC, Chang MS, Hsu MJ, Huang CL, Chen MC, Sheu JR, Chen TF, Chen TL, Inoue H, Lin CH. Bradykinin B2 receptor mediates NF-kappaB activation and cyclooxygenase-2 expression via the Ras/Raf-1/ERK pathway in human airway epithelial cells. J Immunol. 2004 Oct 15;173(8):5219-28. PMID: 15470067.

11. Arcasoy SM, Fisher A, Hachem RR, Scavuzzo M, Ware LB. Report of the ISHLT Working Group on Primary Lung Graft Dysfunction part V: Predictors and outcomes. J Heart Lung Transplant. 2005 Oct;24(10):1483-8. PMID: 16210119.

12. Christie JD, Carby M, Bag R, Corris P, Hertz M, Weill D. Report of the ISHLT Working Group on Primary Lung Graft Dysfunction part II:definition. J Heart Lung Transplant. 2005 Oct;24(10):1454-9. PMID: 16210116

13. Christie JD, Sager JS, Kimmel SE, Ahya VN, Gaughan C, Blumenthal NP, Kotloff RM. Impact of primary graft failure on outcomes following lung transplantation. Chest. 2005 Jan;127(1):161-5. PMID: 15653978.

14. Zhai W, Jungraithmayr W, De Meester I, Inci I, Augustyns $\mathrm{K}$, Arni S, Hillinger S, Scharpé S, Weder W, Korom S. Primary graft dysfunction in lung transplantation: the role of CD26/ dipeptidylpeptidase IV and vasoactiveintestinal peptide. Transplantation. 2009 Apr; 87 (8):1140-6. PMID: 19384159.
15. Jungraithmayr W, De Meester I, Matheeussen V, Inci I, Augustyns K, Scharpé S, Weder W, Korom S. Inhibition of CD26/DPP IV attenuates ischemia/reperfusion injury in orthotopic mouse lung transplants: the pivotal role of vasoactive intestinal peptide. Peptides. 2010 Apr;31(4):585-91.PMID: 20018218.

16. Leeb-Lundberg LM, Marceau F, Müller-Esterl W, Pettibone DJ, Zuraw BL. International union of pharmacology. XLV. Classification of the kinin receptor family: from molecular mechanisms to pathophysiological consequences. Pharmacol Rev. 2005 Mar;57(1):27-77. PMID: 15734727.

17. Ellis KM, Fozard JR. Species differences in bradykinin receptormediated responses of the airways. Auton Autacoid Pharmacol. 2002 Feb;22(1):3-16. PMID: 12423422.

18. Gama Landgraf R, Jancar S, Steil AA, Sirois P. Modulation of allergic and immune complexinduced lung inflammation by bradykinin receptor antagonists. Inflamm Res. 2004 Feb;53(2):78-83. PMID: 15021973.

19. Lambeir AM, Durinx C, Scharpé S, De Meester I. Dipeptidyl-peptidase IV from bench to bedside: an update on structural properties, functions, and clinical aspects of the enzyme DPP IV. Crit Rev Clin Lab Sci. 2003 Jun;40(3):209-94. PMID: 12892317.

\section{Correspondence:}

Prof. Zhiwei Wang

Department of Cardiovascular Surgery

Renmin Hospital, Wuhan University

Jiefang Road 238, Wuchang District, Wuhan 430060, China

wangzhiwei@whu.edu.cn

Received: Aug 10, 2016

Review: Oct 11, 2016

Accepted: Nov 14, 2016

Conflict of interest: none

Financial source: none

${ }^{1}$ Research performed at Department of Cardiovascular Surgery, Renmin Hospital, Wuhan University, Wuhan, China. 